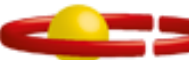 \\ UnC=UE \\ Centro Universitárı de Brasílı
}

CENTRO UNIVERSITÁRIO DE BRASÍLIA - UniCEUB

PROGRAMA DE INICIAÇÃO CIENTÍFICA

CAROLINA FREITAS CARVALHO GUIMARÃES MONTEIRO

LARISSA SOARES DOS SANTOS

PROJETO DE PESQUISAS CLINICAS PARA OS JOGOS PAN AMERICANO DE 2007:

PATOLOGIA DO MANGUITO ROTADOR EM ATLETAS DE ELITE E SUAS ASSOCIAÇÕES COM AMPLITUDE DE MOVIMENTO, FORÇA, DOR E FUNÇÃO DO OMBRO

BRASÍLIA 


\section{$=2$ \\ UnICEUB \\ Centro Unıversıtárıo de Brasílıa}

CAROLINA FREITAS CARVALHO GUIMARÃES MONTEIRO

LARISSA SOARS DOS SANTOS

PROJETO DE PESQUISAS CLINICAS PARA O PAN AMERICANO DE 2007:

PATOLOGIA DO MANGUITO ROTADOR EM ATLETAS DE ELITE E SUAS ASSOCIAÇÕES COM

AMPLITUDE DE MOVIMENTO, FORÇA, DOR E FUNÇÃO DO OMBRO

Relatório final de pesquisa de Iniciação Científica apresentado à Assessoria de Pós-Graduação e Pesquisa.

Orientação: Marcio Oliveira

BRASÍLIA 


\title{
PROJETO DE PESQUISAS CLÍNICAS PARA O PAN AMERICANO DE 2007: PATOLOGIA DO MANGUITO ROTADOR EM ATLETAS DE ELITE E SUAS ASSOCIAÇÕES COM AMPLITUDE DE MOVIMENTO, FORÇA, DOR E FUNÇÃO DO OMBRO
}

\author{
Larissa Soares dos Santos - UniCEUB, PIC HOME, aluno bolsista \\ larissa.soares@sempreceub.com
}

\section{Carolina Freitas Carvalho Guimarães Monteiro - UniCEUB, PIC HOME, aluna voluntária \\ carolina.monteiro30@sempreceub.com}

\begin{abstract}
Marcio Oliveira - UniCEUB, professor orientador
marcio.oliveira@ceub.edu.br
\end{abstract}

\author{
Moacir Silva Neto - Home Ortopedia, orientador \\ moacirsilvaneto@gmail.com
}

A prevalência da lesão do manguito rotador (MR) em geral é alta na população, particularmente em atletas. O objetivo do estudo é determinar a relação entre a patologia do $M R$, avaliada pelo ultrassom e variáveis funcionais, como: amplitude de movimento (ADM), força, dor, função do ombro, medidas antropométricas, incluindo peso, estatura e comprimento do braço de atletas de elite em diferentes esportes. Trata-se de um estudo transversal que investigou 132 atletas de 21 modalidades, participantes do Jogos Panamericanos realizados no Rio de Janeiro em 2007. Todos assinaram o Termo de consentimento livre esclarecido, sendo que o estudo foi aprovado pelo comitê de ética da Universidade de Pittsburgh. Os participantes responderam um questionário geral de saúde que incluíam seus dados demográficos, o questionário 'Incapacidades do Braço, Ombro e Mão' (DASH), e uma Escala Visual Analógica (EVA) para dor nos ombros esquerdo e direito. Além disso, foi realizada uma avaliação ultrassonográfica do ombro dominante. A força dos ombros foi medida com um dinamômetro manual para abdução, rotação interna (RI) e rotação externa (ER) em 0 e 90 graus de abdução, enquanto que a ADM foi determinada por goniometria. A análise estatística foi realizada utilizando o SPSSv.15. A amostra foi dividida em 3 grupos de acordo com os achados da ultrassonografia: grupo 1 (sem achados ultrassonográficos); grupo 2 (apresentaram tendinose e (ou) bursite sub-acromial); grupo 3 (rupturas parciais do MR). Como resultados foram encontrados que atletas com achados ultrassonográficos apresentaram maior prevalência de dor no ombro dominante(DOD) do que aqueles sem qualquer achado $(p=0,040)$. No entanto, $19 \%$ dos atletas com achados ultrassonográficos não relataram DOD. Não houve diferença significativa na prevalência de achados ultrassonográficos entre homens e mulheres $(p=0.700)$. Apesar do grupo 3 reportar uma dor maior na escala visual analógica, $31 \%$ dos atletas com ruptura parcial não reportaram nenhuma dor. Comprimento total do ombro, estatura e idade foram maiores nos grupos com lesão. A pontuação no DASH foi maior no grupo 2, quando comparado ao grupo 1 , sendo que não houve diferença no DASH no grupo 1, em relação ao grupo 3 . Houve maior déficit de força de rotação externa no lado dominante no grupo 3 , comparado ao grupo 1 e 2 . Portanto, foi concluído que a prevalência de lesões do MR em atletas é elevada, muitos deles já com lesões avançadas, não relatam queixa de dor. Existe, também, correlação entre déficit de força de 
rotação externa e rupturas tendineas, além de algumas lesões com as medidas antropométricas, como, estatura, peso e comprimento dos membros superiores.

Palavras-Chave: Lesões em Atletas. Lesões de manguito rotador. Dor. Ultrassonografia. 
LISTA DE TABELAS

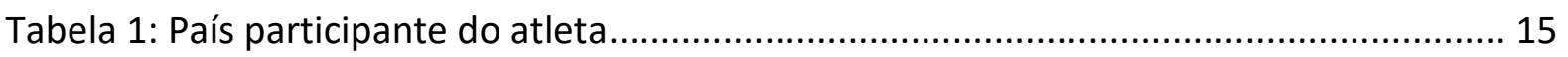

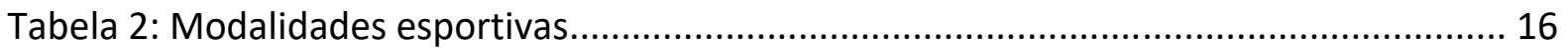

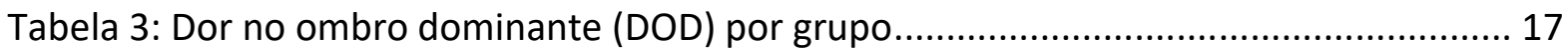

Tabela 4: Medidas Antropométricas por grupo............................................................. 18

Tabela 5: Medidas antropométricas pela presença de dor no ombro no lado dominante (DS) e

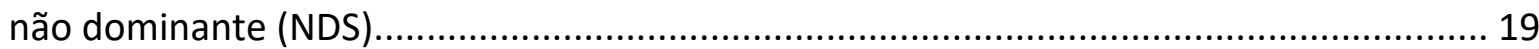

Tabela 6: Escores do DASH, amplitude de movimento (ADM) e força muscular por grupo... 20 Tabela 7: Correlação entre o escore de dor no ombro dominante e razão de torque e

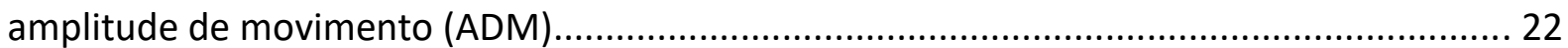

Tabela 8: Relação de amplitude de movimento (ADM) e torque pela presença de dor....... 23 
SUMÁRIO

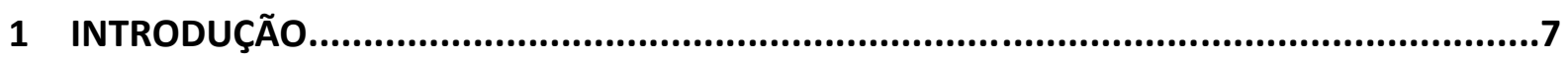

2 FUNDAMENTAÇÃO TEÓRICA

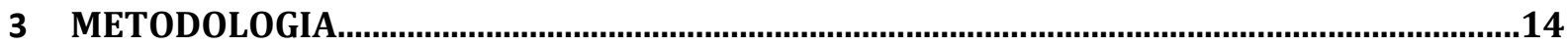

3.1. ANÁLISE DOS DADOS..........................................................................................................

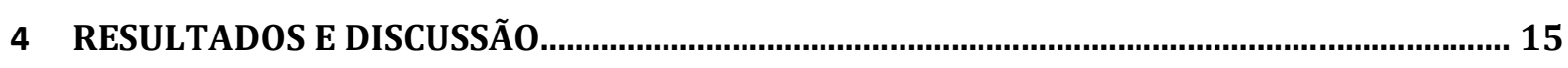

4.1. DESCRIÇÃO DOS ACHADOS DE ULTRASSONOGRAFIA POR

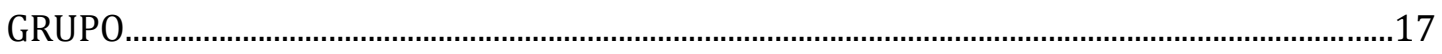

4.2. COMPARAÇÃO DA PRESENÇA DE DOR NO OMBRO DOMINANTE ENTRE OS

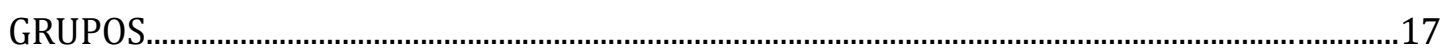

4.3. ACHADOS ULTRASSONOGRÁFICOS E SUA RELAÇÃO COM MEDIDAS

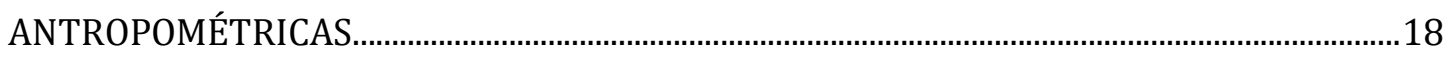

4.4. COMPARAÇÃO DO ESCORE DASH, ADM E FORÇA MUSCULAR ENTRE OS

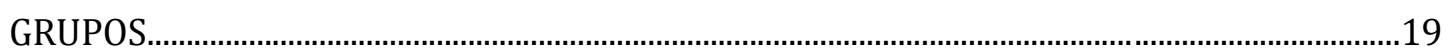

4.5. RELAÇÃO ENTRE DOR NO OMBRO, TORQUE E ADM.............................................................22

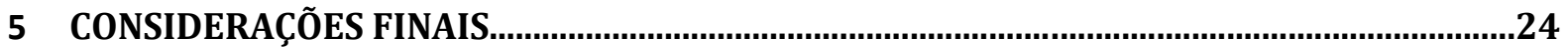

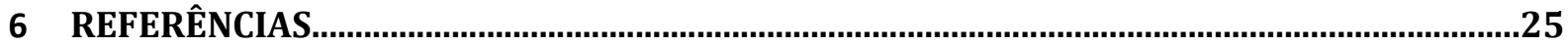

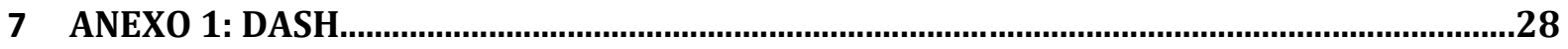

8 ANEXO 2: ESCALA ANALÓGICA DE DOR

9 APÊNDICE A: TERMO DE CONSENTIMENTO

10 APÊNDICCE B: TERMO DE APROVAÇÃO DO CONSELHO DE ÉTICA DA UNIVERSIDADE DE

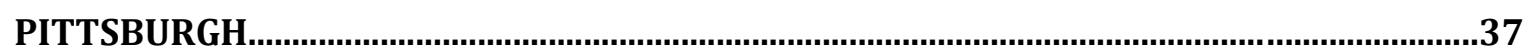




\section{INTRODUÇÃO}

As rupturas totais de manguito rotador estão entre as lesões de ombro mais comuns (COFIELD, 1985), caracterizadas pela dor, fraqueza e diminuição da amplitude de movimento (ADM), além disso, os sintomas geralmente pioram com a atividade, especialmente quando a mão está posicionada sobre a cabeça. A dor noturna também é comum, e muitos se queixam de despertar após rolar sobre o ombro afetado (MURRAY, 2002).

Mais de $80 \%$ dos indivíduos com lesão total dolorosa foram encontrados com deficiência significativa nas atividades da vida diária (AVD), na participação esportiva e nos deveres ocupacionais. Eles achavam que muitas vezes era difícil levantar o braço na altura do ombro para atividades como levantar um recipiente de leite ou pentear o cabelo, sendo que atingir uma prateleira alta era impossível em 55\% das pessoas (WATSON, 2002). Por fim, 50\% dos indivíduos com lesão total de manguito rotador não conseguiram levantar um objeto de 450g para uma prateleira, arremessar, lavar a parte de trás do ombro oposto, ou fazer o trabalho habitual (MATSEN, 1998).

Os sintomas e o prognóstico para a resolução foram atribuídos a muitos fatores, incluindo o tamanho, a espessura, a localização, o número de tendões envolvidos e o tempo da lesão do manguito rotador, bem como a idade do paciente, nível de atividade, dominância das mãos, posição da cabeça humeral na degeneração glenóide e gordurosa dos músculos do manguito rotador (BARTOLOZZI, 1994; BROX, 1996; GOLDBERG, 2001; HARRYMAN, 2003; MATSEN, 1998; MISAMORE, 1995).

Atualmente, um período de descanso, evitando atividades exacerbadas e fazendo reabilitação dos músculos do ombro são recomendadas, por 3 a 6 meses, como tratamento inicial para a maioria das lesões totais de manguito rotador(BARTOLOZZI, 1994; BOKOR, 1993; GREEN, 1998; HAWKINGS, 1995; MANTONE, 2000; WIRTH, 1997). Tais tratamentos tem como objetivo a diminuição da dor e a melhora da ADM do ombro e da força em cerca de $50 \%$ do tempo, tendo enfase no fortalecimento dos músculos circundantes (BARTOLOZZI, 1994; BOKOR, 1993; HAWKINGS, 1995; RUOTOLO, 2002; TYTHERLEIGH-STRONG, 2001; WIRTH, 1997). Se esses tratamentos não cirúrgicos não conseguirem controlar os sintomas do paciente, recomenda-se o reparo cirúrgico da lesão que possui dois propósitos: diminuir não apenas os sintomas da lesão total, mas também minimiza a chance de que a lesão se 
propague para um tamanho maior. Após a cirurgia, a reabilitação dura de seis meses a um ano (GREEN, 1998).

Esse estudo é importante, pois aprofunda sobre a relação da dor e da função em indivíduos com lesão total de manguito rotador que não buscaram tratamento médico para o ombro. Enquanto estudos prévios indicaram que aqueles com uma "assintomática" lesão de seu ombro não eram necessariamente indolores e funcionavam normalmente (CHARD, 1991; YAMAGUCHI, 2001).

Então, não foi projetado para estudar a história natural de lesão total de manguito rotador, que seria demorado, caro e prematuro,e sim, proposto para produzir resultados essenciais para aconselhar indivíduos com lesão total de manguito rotador. Atualmente, o tratamento inicial de indivíduos com lesões dolorosas continua baseado na possibilidade de resolução de sintomas ao longo do tempo. Enquanto um período de descanso, evitando atividades exacerbadas e fazendo reabilitação dos músculos do ombro podem ser efetivo na diminuição dos sintomas em alguns indivíduos com lesão total de manguito rotador, informações precisas do resultado esperado depois permanece deficiente (BARTOLOZZI, 1994; BOKOR, 1993; HAWKINS, 1995; RUOTOLO, 2002; TYTHERLEIGH-STRONG, 2001; WIRTH, 1997).

Enquanto outros questionaram, realizaram exames físicos e obtiveram resultados obtidos em indivíduos com lesão de manguito rotador assintomática, as avaliações abrangentes e quantitativas de ombro no presente estudo forneceram níveis clínicos precisos de dor e função do ombro em indivíduos que não buscaram tratamento médico para lesão total de manguito rotador.

Em alguns indivíduos, a dor de um manguito rotador lesionado persiste muito após a lesão inicial do ombro e é acompanhada por fraqueza e perda de ADM. Em outros, a dor no ombro diminui ao longo do tempo permitindo retornar às AVDs.

Foram estudadas características, cada uma das quais pode ser prontamente determinada em um cenário clínico, que ajudará os médicos a preverem a dor e a função do ombro esperada em qualquer paciente com lesão de manguito rotador. Isto é de imensa importância no tratamento de indivíduos mais velhos com uma dolorosa lesão de manguito rotador do ombro. Ser capaz de identificar características de indivíduos associados a um pior resultado de tratamentos não-cirurgicos diminuirá o desconforto, o tempo de tratamento e os custos. 
Esses indivíduos, susceptíveis de falhar 3 a 6 meses de tratamentos não-cirurgicos, podem escolher tratamentos cirúrgicos logo após a lesão. Por outro lado, a identificação de indivíduos com características associadas a um desfecho favorável de tratamentos nãooperatórios diminuirá a cirurgia desnecessária e os custos associados. Igualmente importantes, as lesões totais de manguito rotador são tão comuns em indivíduos mais velhos que a lesão de ombro pode não ser a etiologia dos sintomas de um paciente. O conhecimento das características de indivíduos com lesão de manguito rotador sem dor possibilitará que os clínicos busquem outras etiologias da dor e da deficiência no ombro do paciente.

Nesse contexto, este estudo possui como objetivo geral identificar características pessoais que são preditivas de sintomas em ombros com lesão total de manguito rotador. Além disso, teve como objetivos específicos: Determinar se indivíduos com lesão total de manguito rotador indolores possuem diferenças na amplitude de movimento, força e resultados funcionais em comparação com indivíduos que têm um manguito rotador intacto e normal ou um manguito rotador doloroso e lesionado; determinar se as diferenças de gênero, etnia, idade contribuem para a presença de dor e deficiência naqueles com lesão total de manguito rotador. 
As rupturas totais do manguito rotador $(M R)$ possuem alta prevalência na população geral, particularmente em idosos e atletas. Esse tipo de lesão é uma causa significativa de incapacidade e tem um impacto muito negativo no estado geral de saúde (GARTSMAN, 1998; MECKEE, 2000). Essa diminuição da qualidade de vida do paciente que apresenta a lesão total do MR está diretamente relacionada com a classificação de gravidade da doença (GOLDBERG, 2001).

Estudos sobre imagens do ombro (CHANDNANI, 1992; GOODMAN, 1995; JOBE, 1994; MILGROM, 1995; MINIACI, 1995; SHER, 1994) e ombros cadavéricos (GRANT, 1948 ; KEUES, 1933; OZAKI, 1988) mostraram que até $39 \%$ dos adultos estudados tinham lesão de manguito de espessura total. No entanto, esses indivíduos geralmente vivem suas vidas com um ombro sem dor e realizam atividades de vida diária (AVDs) sem sintomas. A hipótese é que a ausência de dor pode ser possível se não houver inflamação nas estruturas dentro do ombro e o par de forças entre os músculos do MR anteriores e posteriores estiver preservado (BURKHART, 1992; TIFFORD, 1999).

Estima-se que a prevalência de lesões do MR na população atlética que executa o movimento de sobrecarga seja maior do que na população não atlética, embora dados mais específicos que abordem essa questão ainda sejam necessários. A ruptura total do MR é pouco citada na literatura que inclui atletas de elite, e a maioria de seus dados de incidência são derivados de estudos de imagem.

Um estudo realizado por Connor et al (2003) investigou ombros assintomáticos de atletas (média de 26 anos de idade) de esportes que necessitavam de movimentos aéreos com ressonância magnética (MRI). Eles relataram que 8 dos 20 ombros dominantes (40\%) tiveram achados consistentes com rupturas parcial ou total do MR, em comparação com nenhuma lesão nos ombros não dominantes $(P=0,005)$. Aos 5 anos de acompanhamento, os atletas incluídos naquele estudo não apresentavam nenhum sintoma no ombro dominante.

Segundo a pesquisa feita por Jost et al (2005), foi relatado que 93\% dos jogadores de handebol apresentavam anormalidade no ombro avaliada por ressonância magnética, mas apenas $37 \%$ desses atletas eram sintomáticos. Neste estudo, as lesões MR foram as mais prevalentes, com roturas parciais MR sendo identificadas em $40 \%$ dos casos.

Um estudo sobre a incidência de dor no ombro em tenistas relatou que $24 \%$ dos 270 indivíduos (idade variou entre 12 e 19 anos) queixaram-se de dor no ombro. A incidência de dor no ombro foi maior ( $50 \%$ dos casos) em tenistas de meia-idade (LEHMAN et al, 1988). 
MacMahon et al (2014) investigaram a dor e a função do ombro em 141 atletas de elite com mais de 60 anos e encontraram 21,3\% de atletas com ruptura total do MR. No entanto, apenas $30 \%$ das pessoas com qualquer dano MR relataram dor e $5 \%$ relataram dor, apesar de não ter lesão no MR. O estudo também relatou que a gravidade da lesão do MR não foi associada à dor.

Parece que a lesão nem sempre é acompanhada de dor. Então, ao examinar ombros indolores, não se pode descartar a presença de uma lesão. Estudos mostraram que até $40 \%$ dos lançadores com ombros assintomáticos já apresentam rupturas parciais ou completas do MR (JOST, 2005). Além disso, ao investigar déficits de ADM e desequilíbrios de força do ombro em atletas, deve-se estar ciente de que atletas de elite são capazes de se adaptar à lesão para que possam continuar a ter um bom desempenho em seu esporte específico. Essa é uma característica que diferencia esses indivíduos da população não atlética.

Vários estudos descreveram uma faixa de valores para déficits de ADM do ombro e desequilíbrios de força em atletas saudáveis que diferem de acordo com os esportes que praticam (BORSA, 2005; PRICKETT, 2003; SHER, 1994; ELLENBECKER, 2002). Portanto, avaliar as associações dos déficits da ADM do ombro e desequilíbrios de força com a lesão do ombro nesses atletas é problemático, uma vez que o próprio esporte, e não a lesão, pode ser responsável pela presença de déficits de ADM e desequilíbrios de força.

Até o momento, não se sabe se os atletas com lesões assintomáticas de MR serão mais propensos a futuros sintomas e disfunções do ombro do que aqueles com tendões do MR normais.

\section{METODOLOGIA}

Trata-se de um estudo transversal que investigou as condições do ombro de atletas participantes dos Jogos Pan-Americanos realizados no Rio de Janeiro (RJ) em 2007. Uma 
equipe de pesquisadores composta por médicos, estudantes de medicina, fisioterapeutas e estudantes de fisioterapia trabalharam em torno do local do Pan-Americano. Atletas de vários esportes e países foram convidados a participar como voluntários no nosso estudo. Todos os atletas de 18 anos ou mais que aceitaram participar do estudo consentiram com os procedimentos do estudo e assinaram um termo de consentimento. $O$ estudo de pesquisa e o termo de consentimento (Apêndice A) foram aprovados pelo comite de ética da Universidade de Pittsburgh (Apêndice B).

Os atletas participantes completaram um questionário geral de saúde que incluía seus dados demográficos, o questionário 'Incapacidades do Braço, Ombro e Mão' (DASH) (Anexo 1 - DASH), que é um questionário de auto-relatório de 30 itens, projetado para medir sintomas e função em indivíduos com distúrbios músculo-esqueléticos do membro superior.

Além disso, foram classificados pela Escala Visual Analógica (EVA) para dor nos ombros esquerdo e direito (o atleta marcou seu nível de dor em uma linha de $10 \mathrm{~cm}$, em que a posição marcada correspondia a um escore entre 0 e 100: onde o zero não indicava dor e 100 a dor máxima possível). Os sujeitos que classificaram sua dor como "sem dor" ou menos de $10 \mathrm{~mm}$ no VAS foram considerados como tendo um ombro indolor. Os ombros dolorosos foram os sujeitos que avaliaram sua dor de 10 a 100 na VAS. A presença de dor foi determinada a partir do escore EVA e de uma questão simples sobre se tinham ou não dor no ombro (sim / não).

Depois de completar os questionários, dois testadores mediram a ADM do ombro esquerdo e direito dos atletas. Enquanto um testador estabilizou o ombro, o outro usou um goniômetro padrão para medir a ADM de flexão do ombro, rotação externa a $0^{\circ}$ (ER 0) e $90^{\circ}$ (ER 90) de abdução do ombro e rotação interna a $90^{\circ}$ de abdução do ombro (IR 90). Essas medidas foram utilizadas para determinar uma relação da ADM (ADMR): ADM do ombro dominante (DS) dividido pela ADM do ombro não dominante (NDS). Todos os testadores foram treinados por pessoal sênior antes de realizar as medições.

A amplitude de movimento ativa foi determinada por: elevar os braços sobre a cabeça, colocar as mãos atrás da cabeça e espalhar os cotovelos, alcançar o topo do ombro oposto e chegar atrás das costas. Cada movimento será classificado de acordo com os critérios estabelecidos.

Após as medições da ADM, os testadores avaliaram a força do ombro dos atletas em ambos os lados usando um dinamômetro manual (Lafayette Manual Muscle Tester; Modelo 01163; Lafayette, IN). Força isométrica de abdutores de ombro e rotadores internos e 
externos (a $0^{\circ}$ [IR 0 e ER 0] e a $90^{\circ}$ de abdução de ombro [IR 90 e IR 90]) foram avaliados usando posições padronizadas. Os atletas foram instruídos a exercer o esforço máximo em cada posição do ombro, além de expirar para evitar a manobra de valsalva.

Os valores de torque muscular foram descritos em $\mathrm{kg} . \mathrm{cm}$ usando força (Kg), indicada pelo dinamômetro, e o braço de alavanca $(\mathrm{cm})$, que é a distância medida entre o eixo de rotação (ponto médio do ombro) e o ponto em que o dinamômetro foi colocado. Para abdução, multiplicamos a força pelo comprimento da parte superior do braço do acromio até o ponto de aplicação do dinamômetro manual apenas proximal ao epicôndilo lateral do úmero. Para a rotação interna e externa, multiplicamos a força pelo comprimento do antebraço medido do epicôndilo lateral até o ponto de aplicação do dinamômetro de mão apenas proximal ao estilóide ulnar. A produção de torque foi expressa em relação ao peso corporal.

As relações de torque foram determinadas pela divisão do torque do DS pelo torque do NDS. Além disso, as razões de torque entre os músculos agonistas-antagonistas foram calculadas para IR / ER em 0 e 90 graus de abdução do ombro foram calculados: torque de IR pelo torque ER.

Procedimentos de medição e auto-relatórios foram realizados e registrados em todos os assuntos antes de completarem a avaliação da dor VAS e o ultra-som. O pesquisador e os fisioterapeutas que realizaram os procedimentos de medição foram cegados a esta informação. O coordenador do estudo não estava envolvido na realização de nenhum dos procedimentos de medição.

Para avaliar lesões musculoesqueléticas do ombro, quatro radiologistas treinados avaliaram o DS dos atletas por meio de ultrassonografia. Os radiologistas usaram o mesmo protocolo para identificar bursite sub-acromial, tendinose no MR e ruptura parcial / completa no ombro. Este tipo de avaliação ultra-sonográfica mostrou alta especificidade (96-98\%) e sensibilidade (80-94\%) no diagnóstico de ruptura parcial do MR, (OZAKI, 1988; CONNOR, 2003; BORSA, 2005; PRICKETT, 2003; SHER, 1994) que não difere da especificidade e sensibilidade relatadas no diagnóstico de lesão parcial do MR usando ressonância magnética (LENZA, 2013).

Os achados ultra-sonográficos foram divididos em 3 grupos diferentes da seguinte forma:

- Grupo 1 composto por indivíduos que não tiveram nenhum achado ultrassonográfico;

- Grupo 2 composto por aqueles que tiveram tendinose e / ou bursite sub-acromial; 
- Grupo 3 composto por aqueles que tiveram rupturas parciais ou totais do MR (com ou sem outros achados).

\subsection{Análise de dados}

A estatística descritiva das variáveis contínuas incluiu média e desvio-padrão ou mediana e percentis $25-75$, de acordo com a distribuição dos dados. Contagens e freqüências foram usadas para variáveis categóricas. A normalidade dos dados foi avaliada pelo teste de Shapiro-Wilk.

O teste de Kruskal-Wallis $\mathrm{H}$ foi utilizado para analisar a relação entre os três grupos de ultrassom e as variáveis contínuas avaliadas (ou seja, nível de dor, ADM, força, idade, comprimento do braço, escores do DASH). Os testes do qui-quadrado foram usados para analisar a relação entre os grupos de ultrassom e as variáveis categóricas (ou seja, dor versus ausência de dor). A correlação de Pearson foi usada para examinar a correlação entre a intensidade da dor e os resultados funcionais. A análise estatística foi realizada utilizando o SPSS V.15.

\section{RESULTADOS E DISCUSSÃO}

Cento e trinta e dois atletas de 24 países (Tabela 1) e 21 Modalidades Esportivas (Tabela 2) participaram do estudo. Desses, pudemos avaliar 90 (68,2\%) usando o ultrassom. Dos 132 participantes, 45,1\% eram do sexo feminino, com média de idade de 27,0 $(8,1)$ anos, altura de $177,0(10,5) \mathrm{cm}$ e peso de $79,5(22,2) \mathrm{kg}$.

Tabela 1. País participante do atleta 


\begin{tabular}{|c|c|c|}
\hline & Frequência & Porcentagem \\
\hline Antilhas & & \\
\hline Holandesas & & 0.76 \\
\hline Argentina & 4 & 3.03 \\
\hline Aruba & 1 & 0.76 \\
\hline Bahamas & 1 & 0.76 \\
\hline Barbados & 5 & 3.79 \\
\hline Belize & 1 & 0.76 \\
\hline Brasil & 19 & 14.39 \\
\hline Canadá & 5 & 3.79 \\
\hline Chile & 2 & 1.52 \\
\hline Dominica & 2 & 1.52 \\
\hline Equador & 5 & 3.79 \\
\hline Estados Unidos & 2 & 1.52 \\
\hline El Salvador & 3 & 2.27 \\
\hline Jamaica & 2 & 1.52 \\
\hline México & 16 & 12.12 \\
\hline Nicarágua & 1 & 0.76 \\
\hline Panamá & 11 & 8.33 \\
\hline Peru & 3 & 2.27 \\
\hline Porto Rico & 1 & 0.76 \\
\hline República & 14 & \\
\hline Dominicana & & 10.61 \\
\hline Santa Lucia & 1 & 0.76 \\
\hline Trinidad e Tobago & 4 & 3.03 \\
\hline Uruguai & 18 & 13.64 \\
\hline Venezuela & 1 & 0.76 \\
\hline Não respondeu & 9 & 6.82 \\
\hline Total & 132 & 100.0 \\
\hline
\end{tabular}

Tabela 2. Modalidades esportivas 


\begin{tabular}{|c|c|c|}
\hline & Frequência & Porcentagem \\
\hline Tiro com arco & 10 & 7.58 \\
\hline Badminton & 1 & 0.76 \\
\hline Beisebol & 5 & 3.79 \\
\hline Basquete & 3 & 2.27 \\
\hline Boliche & 1 & 0.76 \\
\hline Boxe & 1 & 0.76 \\
\hline Canoagem/caiaque & 7 & 5.30 \\
\hline Hóquei em campo & 18 & 13.64 \\
\hline Judô & 5 & 3.79 \\
\hline Karatê & 1 & 0.76 \\
\hline Remo & 6 & 4.55 \\
\hline Tiro & 5 & 3.79 \\
\hline Squash & 2 & 1.52 \\
\hline Natação & 6 & 4.55 \\
\hline Tênis de mesa & 1 & 0.76 \\
\hline Taekwondo & 2 & 1.52 \\
\hline Handebol & 16 & 12.12 \\
\hline Vôlei & 11 & 8.33 \\
\hline Vôlei de praia & 2 & 1.52 \\
\hline Polo aquático & 5 & 3.79 \\
\hline Levantamento de peso & 4 & 3.03 \\
\hline Corrida de 100 metros & 1 & 0.76 \\
\hline Salto triplo & 1 & 0.76 \\
\hline Arremesso de peso & 5 & 3.79 \\
\hline Arremesso de dardo & 2 & 1.52 \\
\hline Heptatlo & 1 & 0.76 \\
\hline Corrida de 200 metros & 1 & 0.76 \\
\hline Não respondeu & 8 & 6.06 \\
\hline Total & 132 & 100.0 \\
\hline
\end{tabular}

\subsection{Descrição dos achados de Ultrassonografia por Grupo}

De todos os atletas avaliados por Ultrassonografia $(n=90)$ identificamos o seguinte: 
- 41 indivíduos $(45,6 \%)$ tinham "Sem achados ultrassonográficos" (Grupo 1)

- 34 indivíduos (37,8\%) tinham "Tendinose ou Bursite" (Grupo 2)

- 15 indivíduos (16,7\%) tinham 'ruptura parcial do manguito rotador' (Grupo 3)

- Nenhum tinha ruptura completa do MR.

- Todas as rupturas parciais de MR estavam no músculo supraespinhal.

Atletas com quaisquer achados ultrassonográficos apresentaram maior prevalência de dor no ombro dominante do que aqueles sem qualquer achado $(p=0,040)$. No entanto, $19 \%$ dos atletas com achados ultrassonográficos não relataram dor no ombro avaliado. Aqueles com ruptura parcial do MR (31\%) não relataram nenhuma dor no ombro dominante. Não houve diferença significativa na prevalência de achados ultrassonográficos entre homens e mulheres $(p=0.700)$.

\subsection{Comparação da presença de dor no ombro dominante entre os grupos}

A avaliação cruzada utilizando o teste Qui Quadrado mostrou uma relação positiva entre os grupos de ultrassonografia e uma dor no ombro dominante (relatada pelo atleta como dolorosa ou não dolorosa). Como esperado, ombros dolorosos foram mais prevalentes no Grupo 3, seguido pelo Grupo 2 e Grupo 1 (Tabela 3).

Tabela 3. Dor no ombro dominante (DOD) por grupo

\begin{tabular}{lllll}
\hline & & Group 1 & Group 2 & Group 3 \\
\hline DOD & No pain & $25(60.97 \%)$ & $11(32.35 \%)$ & $4(26.67 \%)$ \\
& Painful & $16(39.02 \%)$ & $23(67.65 \%)$ & $11(73.33 \%)$ \\
& Total & $41(100.00 \%)$ & $34(100.00 \%)$ & $15(100.00 \%)$ \\
\hline
\end{tabular}

$\mathrm{p}=.040$

O escore de dor no ombro dominante medido pela EVA foi maior no Grupo 3 em comparação ao Grupo $1(27,5$ [32,3] vs. 9,2 [18,5]; $p=0,016)$ e maior no Grupo 2 em comparação ao Grupo 1 (17,5 [24,8] vs. 9,2 [18,5]; $p=0,039)$. O Grupo 3 também teve um escore EVA maior do que o Grupo 2, mas essa diferença não foi estatisticamente significativa $(27,5[32,3]$ vs. $17,5[24,8] ; p=.300)$.

Enquanto todos os atletas competiam ativamente nos jogos pan-americanos, $11,4 \%$ deles apresentavam ruptura parcial do manguito rotador. A patologia do manguito rotador foi associada à dor, mas um achado clinicamente relevante foi que $26,7 \%$ dos atletas com lesões parciais não relataram dor em seu ombro dominante. Esse achado concorda com um 
estudo em atletas mais velhos (> 60 anos de idade) que mostrou que $68 \%$ dos atletas com lesão parcial do manguito rotador não relataram dor (MCMAHON, 2014).

Outro estudo sobre lesão parcial do manguito rotador descobriu que atletas que tiveram ombros assintomáticos permaneceram sem sintomas por 5 anos sem tratamento (CONNOR, 2003). A dor é conhecida como um sintoma importante que leva à investigação da patologia, no entanto, estudos mostraram que algumas lesões do ombro podem não ser sintomáticas. Assim, a dor não deve ser o único critério usado na investigação de lesões do ombro relacionadas ao esporte.

\subsection{Achados ultrassonográficos e sua relação com medidas antropométricas}

A Tabela 4 mostra a estatística descritiva para idade, peso, altura e comprimento total do braço (TAL) nos Grupos 1, 2 e 3. A análise estatística mostrou que o Grupo 1 era mais jovem em comparação ao Grupo 2 ( $p=0,001)$ e Grupo $3(p=0,001)$. Os atletas do Grupo 1 também eram mais magros que os do Grupo $2(p=0,003)$, menores que os do Grupo $3(p=0,016)$, e TAL mais curto que os do Grupo $3(p=0,024)$. Não houve diferenças significativas entre os grupos para as outras comparações.

Tabela 4. Medidas Antropométricas por grupo

\begin{tabular}{lllll}
\hline & Grupo 1 & Grupo 2 & Grupo 3 & Valores de p \\
Idade (anos) & $23.48(4.96)$ & $29.72(9.68)$ & $31.50(9.59)$ & $\mathbf{. 0 0 1 a} / .001 \mathbf{b} / .400 \mathrm{c}$ \\
Peso (Kg) & $73.70(18.87)$ & $87.14(23.75)$ & $81.89(16.52)$ & $.070 \mathrm{a} / .003 \mathbf{b} / .490 \mathrm{c}$ \\
Altura (cm) & $174.23(10.73)$ & $179.10(9.20)$ & $183.25(9.65)$ & $\mathbf{. 0 1 6 a} / .065 \mathrm{~b} / .086 \mathrm{c}$ \\
TAL (cm) & $102.90(8.38)$ & $105.70(11.39)$ & $108.00(8.94)$ & $\mathbf{. 0 2 4 a} / .100 \mathrm{~b} / .200 \mathrm{c}$ \\
\hline
\end{tabular}

a- Comparação entre o Grupo 1 e o Grupo 3

b- Comparação entre o grupo 1 e o grupo 2

c- Comparação entre o Grupo 2 e o Grupo 3

A Tabela 5 mostra a estatística descritiva para idade, peso, altura e TAL em atletas que relataram dor e sem dor no ombro dominante (SD). Não houve diferenças entre os grupos com relação às variáveis antropométricas em atletas com ou sem dor no ombro.

Tabela 5. Medidas antropométricas pela presença de dor no ombro no lado dominante (DS) e não dominante (NDS)

\begin{tabular}{llll}
\hline & DS com dor & DS sem dor & Valor de p \\
Idade (anos) & $25.84(5.29)$ & $27.00(8.32)$ & .440 \\
Peso (Kg) & $80.64(20.22)$ & $79.00(23.59)$ & .722 \\
\hline
\end{tabular}




\begin{tabular}{llll}
\hline Altura $(\mathrm{cm})$ & $177.86(9.39)$ & $176.42(10.72)$ & .502 \\
TAL $(\mathrm{cm})$ & $105.55(7.70)$ & $104.52(9.80)$ & .691
\end{tabular}

A idade tem sido claramente associada à patologia do manguito rotador na literatura e verificou-se ter a mesma associação neste estudo. Diferentemente da população não atlética em que as rupturas parciais do manguito rotador estão presentes em idade mais avançada, a amostra de atletas de elite com a lesão deste estudo foi de meia-idade (média de 31 anos de idade). A ocorrência de lesões do manguito rotador em adultos jovens é provavelmente devido ao uso excessivo das extremidades superiores, uma vez que eram todos atletas de elite. O peso corporal foi maior naqueles com tendinose / bursite (Grupo 2) no ombro, mas não encontramos uma explicação plausível para esse achado.

Indivíduos mais altos e com braços mais longos apresentaram maior incidência de rupturas parciais do manguito rotador, provavelmente devido ao braço de alavanca mais longo que pode gerar mais estresse nas estruturas estabilizadoras (por exemplo, tendões do manguito rotador) no ombro ao realizar atividades aéreas em comparação com braços de alavanca mais curtos.

\subsection{Comparação do escore DASH, ADM e força muscular entre os grupos}

A Tabela 6 apresenta a estatística descritiva para os escores do Dash (escore total e escore específico do esporte), valores da ADM, relação da ADM entre ombro dominante (DS) e ombro não dominante (NDS), valores de torque e relação de torque entre DS e NDS. Houve poucas diferenças significativas encontradas quando comparamos os escores entre os grupos de ultrassonografia (Tabela 6). O Grupo 2 teve maiores escores do Dash Total e do Esporte em comparação ao Grupo 1 ( $p=0,035$ e $p=0,002$, respectivamente). O grupo 2 também apresentou uma maior razão de ADM (DS / NDS) do que o Grupo 1 para rotação total (ou seja, IR90 + ER90) $(p=0$,019). Para relação de torque muscular (DS / NDS), o Grupo 1 apresentou maior razão de torque para IR0 do que para o Grupo 2 ( $p=0,016)$, o Grupo 3 apresentou menor razão de torque para ER0 em relação ao Grupo $2(p=0,043)$ e Grupo $1(p=0,007)$. Grupo 3 também apresentou uma menor relação de torque para o ER90 do que o Grupo 2 ( $p$ $=0,031)$ e o Grupo $1(p=0,008)$. Todas as outras comparações entre grupos não foram estatisticamente significativas.

Tabela 6. Escores do DASH, amplitude de movimento (ADM) e força muscular por grupo 


\begin{tabular}{|c|c|c|c|c|}
\hline & Grupo 1 & Grupo 2 & Grupo 3 & Valores de p \\
\hline \multicolumn{5}{|l|}{ DASH } \\
\hline Pontuação Total & $5.44(7.60)$ & $10.24(11.10)$ & $5.38(6.40)$ & $.400 \mathrm{a} / .035 b / .220 \mathrm{c}$ \\
\hline Pontuação & $7.81(15.00)$ & $21.06(20.80)$ & $12.94(17.90)$ & $.100 \mathrm{a} / .002 \mathrm{~b} / .240 \mathrm{c}$ \\
\hline \multicolumn{5}{|l|}{ esportiva } \\
\hline \multicolumn{5}{|l|}{ ADM } \\
\hline Flexão & $171.5(7.02)$ & $166.70(14.40)$ & $169.20(9.60)$ & $.500 \mathrm{a} / .300 \mathrm{~b} / .700 \mathrm{c}$ \\
\hline ER 0 & $63.10(16.00)$ & $60.00(14.20)$ & $58.70(9.00)$ & $.400 \mathrm{a} / .400 \mathrm{~b} / .700 \mathrm{c}$ \\
\hline IR 90 & $58.30(16.40)$ & $57.50(15.19)$ & $52.17(15.70)$ & $.200 \mathrm{a} / .700 \mathrm{~b} / .300 \mathrm{c}$ \\
\hline ER 90 & $88.50(12.10)$ & $84.70(16.55)$ & $89.42(10.90)$ & $.800 a / .100 b / .300 c$ \\
\hline Rotação Total & $146.81(21.80)$ & $141.80(22.40)$ & $141.60(19.90)$ & $.600 \mathrm{a} / .500 \mathrm{~b} / .800 \mathrm{c}$ \\
\hline \multicolumn{5}{|l|}{ Relação ADM } \\
\hline Flexão & $1.00(0.03)$ & $0.99(0.07)$ & $1.01(0.05)$ & $.300 \mathrm{a} / .900 \mathrm{~b} / .300 \mathrm{c}$ \\
\hline ER 0 & $1.02(0.21)$ & $1.10(0.26)$ & $1.05(0.11)$ & $.200 \mathrm{a} / .100 \mathrm{~b} / .700 \mathrm{c}$ \\
\hline IR 90 & $0.90(0.19)$ & $0.97(0.21)$ & $0.91(0.20)$ & $.500 \mathrm{a} / .300 \mathrm{~b} / .300 \mathrm{c}$ \\
\hline ER 90 & $1.04(0.09)$ & $1.10(0.19)$ & $1.05(0.09)$ & $.400 \mathrm{a} / .200 \mathrm{~b} / .800 \mathrm{c}$ \\
\hline Rotação Total & $0.97(0.09)$ & $1.03(0.11)$ & $0.96(0.10)$ & $.800 \mathrm{a} / .019 b / .100 \mathrm{c}$ \\
\hline \multicolumn{5}{|l|}{ Torque } \\
\hline Abdução & $47.42(17.24)$ & $50.68(20.70)$ & $44.74(13.89)$ & $.600 \mathrm{a} / .700 \mathrm{~b} / .300 \mathrm{c}$ \\
\hline IR 0 & $42.68(18.37)$ & $41.98(14.28)$ & $39.62(15.23)$ & $.600 \mathrm{a} / .700 \mathrm{~b} / .700 \mathrm{c}$ \\
\hline ER 0 & $37.01(14.21)$ & $38.85(14.87)$ & $34.12(12.21)$ & $.400 \mathrm{a} / .700 \mathrm{~b} / .400 \mathrm{c}$ \\
\hline IR 90 & $42.79(18.36)$ & $42.56(13.84)$ & $41.32(15.53)$ & $.800 \mathrm{a} / .700 \mathrm{~b} / .800 \mathrm{c}$ \\
\hline ER 90 & $41.20(14.44)$ & $42.21(15.75)$ & $36.30(16.08)$ & $.300 \mathrm{a} / .700 \mathrm{~b} / .300 \mathrm{c}$ \\
\hline \multicolumn{5}{|l|}{ Relação Torque } \\
\hline Abdução & $0.81(0.28)$ & $0.81(0.25)$ & $0.74(0.22)$ & $.100 \mathrm{a} / .600 \mathrm{~b} / .300 \mathrm{c}$ \\
\hline IR 0 & $1.10(0.17)$ & $0.97(0.18)$ & $1.01(0.26)$ & $.053 a / .016 b / .900 c$ \\
\hline ER 0 & $1.05(0.16)$ & $1.02(0.24)$ & $0.89(0.16)$ & $.007 a / .500 b / .043 c$ \\
\hline IR 90 & $1.00(0.16)$ & $1.01(0.20)$ & $1.02(0.32)$ & $.600 \mathrm{a} / .900 \mathrm{~b} / .600 \mathrm{c}$ \\
\hline ER 90 & $1.04(0.18)$ & $1.03(0.26)$ & $0.80(0.26)$ & $.008 a / .700 b / .031 c$ \\
\hline Relação IR/ER 0 & $1.06(0.20)$ & $0.98(0.22)$ & $1.15(0.30)$ & $.600 \mathrm{a} / .060 \mathrm{~b} / .066 \mathrm{c}$ \\
\hline Relação IR/ER & $0.99(0.23)$ & $1.03(0.31)$ & $1.47(1.06)$ & $.089 a / .900 b / .104 c$ \\
\hline 90 & & & & \\
\hline
\end{tabular}




$$
\begin{aligned}
& \text { a- Comparação entre o Grupo } 1 \text { e o Grupo } 3 \\
& \text { b- Comparação entre o grupo } 1 \text { e o grupo } 2 \\
& \text { c- Comparação entre o Grupo } 2 \text { e o Grupo } 3
\end{aligned}
$$

Acredita-se que a cronicidade pode ter desempenhado um papel na comparação entre as medidas da ADM e os achados ultrassonográficos. A relação ADM foi diferente entre aqueles sem nenhum achado ultra-sonográfico (Grupo 1) e aqueles com tendinose / bursite (Grupo 2), mas não entre os Grupos 1 e 3 (atletas com ruptura parcial do manguito rotador). A tendinose / bursite é uma lesão mais aguda, que pode ser mais dolorosa e limitar a capacidade do indivíduo de atingir a ADM completa. Por outro lado, as rupturas do manguito rotador podem ser mais crônicas e o ombro pode ter se adaptado à lesão, o que pode não ser mais um fator limitante (ZANCA, 2011). Embora se possa esperar que indivíduos com lesão do ombro apresentem uma ADM limitada, em comparação àqueles sem lesão, isso pode ser verdade apenas quando a lesão do ombro está em fase aguda / subaguda em atletas de elite.

Ao comparar a força dos músculos do ombro entre os grupos, aqueles com ruptura parcial do manguito apresentaram déficits de rotação externa em 0 e 90 graus de abdução em comparação aos grupos 1 e 2, enquanto não houve diferenças entre os grupos 1 e 2. Os achados concordam com os achados de Johansson et al sobre tenistas de elite (JOHNSSON, 2015). Portanto, uma lesão mais grave, como a ruptura do manguito rotador, parece levar à fraqueza muscular. Embora tenha sido identificado todas as rupturas parciais no supraespinhoso, que é um rotador interno, a força de rotação externa foi prejudicada nesses atletas.

A fraqueza da rotação externa pode ter causado a patologia do manguito rotador e não o contrário. Esta é uma justificativa lógica, pois o supra-espinhal não é responsável pela rotação externa a 0 grau de abdução do ombro, e sua lesão isolada não deve diminuir a força da rotação externa. Outra explicação para esse achado é uma disfunção na teoria da relação força-acoplamento do manguito rotador, que levaria a uma migração superior da cabeça do úmero na fossa glenoide, colidindo com o supraespinhal no arco coracoacromial.

Outros estudos mostraram que a rotação interna também está comprometida nesses casos. No presente estudo, a força de rotação interna foi menor no Grupo 3, mas não foi diferente entre os grupos, nem estatisticamente ou clinicamente. A rotação externa, por outro lado, mostrou relação clínica e estatística com rupturas parciais do manguito rotador.

\subsection{Relação entre dor no ombro e torque e ADM}


A Tabela 7 mostra os coeficientes de correlação de Pearson entre o escore de dor (EVA) no ombro dominante e o torque e ADM. Os escores de dor mostraram correlação positiva com a relação agonista-antagonista (IR / ER) em 0 e 90 graus de abdução do ombro, indicando que maiores valores da relação IR/ER (ou seja, maior saldo muscular) levaram a maiores escores de dor VAS.

Tabela 7. Correlação entre o escore de dor no ombro dominante e razão de torque e amplitude de movimento (ADM)

\begin{tabular}{lll}
\hline & Correlação & Valor de \\
Torque & Pearson & $\mathbf{p}$ \\
Abdução & -0.09 & .369 \\
IR 0 & -0.04 & .703 \\
ER 0 & -0.19 & .054 \\
IR 90 & 0.18 & .071 \\
Relação IR/ER 0 & 0.21 &. $\mathbf{0 3 8}$ \\
Relação IR/ER 90 & 0.32 &. $\mathbf{0 0 1}$ \\
ADM & & \\
ER 0 & 0.03 & .772 \\
IR 90 & 0.02 & .830 \\
ER 90 & -0.11 & .280 \\
Flexão & 0.07 & .501 \\
Rotação total & -0.06 & .592 \\
\hline
\end{tabular}

A Tabela 8 mostra os descritivos para as razões ADM e Torque (DS / NDS) nos grupos que relataram dor e sem dor. Não houve diferenças estatisticamente significantes relacionadas à $A D M$ e razão de torque entre os grupos com dor e sem dor no ombro dominante.

Tabela 8. Relação de amplitude de movimento (ROM) e torque pela presença de dor

\begin{tabular}{|c|c|c|c|}
\hline Relação ADM & $\begin{array}{l}\text { DS com } \\
\text { dor }\end{array}$ & $\begin{array}{l}\text { NDS com } \\
\text { dor }\end{array}$ & $\begin{array}{l}\text { Valor de } \\
\text { p }\end{array}$ \\
\hline ER 0 & $1.06(0.19)$ & $1.07(0.24)$ & .960 \\
\hline IR 90 & $0.88(0.26)$ & $0.90(0.20)$ & .859 \\
\hline ER 90 & $1.04(0.13)$ & $1.07(0.13)$ & .829 \\
\hline
\end{tabular}




\begin{tabular}{llll}
\hline Flexão & $1.00(0.05)$ & $0.98(0.08)$ & .106 \\
Rotação Total & $0.96(0.11)$ & $0.99(0.13)$ & .573 \\
Relação Torque & & & \\
Abdução & $0.78(0.21)$ & $0.80(0.21)$ & .474 \\
ER 90 & $0.99(0.28)$ & $1.00(0.18)$ & .769 \\
IR 90 & $1.03(0.25)$ & $0.97(0.16)$ & .587 \\
IR 0 & $1.01(0.23)$ & $1.04(0.16)$ & .140 \\
ER 0 & $0.98(0.17)$ & $1.02(0.20)$ & .330 \\
Relação IR/ER 0 & $1.08(0.38)$ & $1.04(0.19)$ & .684 \\
Relação IR/ER 90 & $1.17(0.69)$ & $1.00(0.22)$ & .694 \\
\hline
\end{tabular}

As lesões do manguito rotador foram relacionadas a vários parâmetros estudados, incluindo a dor. No entanto, ao comparar atletas que tiveram dor no ombro com aqueles que não a tiveram, não houve diferenças na ADM e no torque. As associações entre a intensidade da dor e a relação agonista-antagonista indicaram que uma maior proporção leva a maiores escores de dor. Uma possível explicação para esse achado é que a relação agonistaantagonista aumentada pode estar levando a um aumento na pressão articular ou na rigidez muscular, o que pode estar aumentando o desconforto / dor no ombro.

\section{CONSIDERAÇÕES FINAIS}

A patologia do manguito rotador é muito comum na população atlética, mesmo naqueles que não relatam dor. Essas lesões, mesmo quando não sintomáticas, podem causar déficits de força e função. O objetivo de todos os médicos deve ser não apenas tratar essas lesões, mas também impedir que elas aconteçam.

A análise de força via dinamometria manual é um método simples e barato que pode identificar desequilíbrios que podem estar relacionados à patologia do manguito rotador. Este estudo avaliou como variáveis funcionais poderiam estar ligadas à patologia do manguito rotador em atletas de elite. Os atletas estudados eram de vários esportes e países, o que deu 
um amplo espectro do efeito do gesto esportivo específico na amplitude de movimento do ombro e no equilíbrio de força.

Outra vantagem deste estudo foi que a maioria dos atletas teve seu ombro avaliado por ultrassonografia para identificar a presença de patologia do manguito rotador, apesar da presença de dor. Geralmente, atletas sem dor não participam de estudos que envolvam a investigação de patologias do ombro.

Portanto, o estudo não foi desenvolvido para abordar a incidência da patologia do manguito rotador porque todos os atletas envolvidos estavam participando de um evento esportivo de alto nível, e aqueles que têm ferimentos graves são normalmente impedidos de participar de tais eventos.

Estudos longitudinais adicionais ainda são necessários para abordar quando os desequilíbrios de força podem ser a causa ou apenas as consequências da patologia do manguito rotador.

\section{REFERÊNCIAS}

BARTOLOZZI, A.; ANDREYCHIK, D.; AHMAD, S. Determinants of outcome in the treatment of rotador cuff disease. Clin Orthop, 308: 90-97, 1994.

BOKOR, D. J.; HAWKINS, R. J.; HUCKELL, G. H. Results of nonoperative management of fullthockeness tears of the rotator cuff. Clin Orthop, 294: 103-110, 1993

BORSA, P. A.; WILK, K. E.; JACOBSON, J. A.; SCIBEK, J. S.; DOVER, G. C.; REINOLD, M. M.;

ANDREWS, J. R. Correlation of Range of Motion and Glenohumeral Translation in Professional Baseball Pitchers. The American Journal of Sports Medicine, Vol. 33, No. 9 :1392-1399, 2005.

BROX, J.I.; BREVIK, J. I.; LHUNGGREN, A. E.; STAFF, P. H. Influence of anthropometric and psychosocial variables, pain and disability on isometric endurance of shoulder abduction in patientes with rotator tendinosis of the sholder. Scan J Rehab Med, 28: 193-200, 1996. 
BURKHART, S. S. Fluoroscopic comparison of kinematic patterns in massive rotator cuff tears: A suspension bridge model. Clin Orthop, 284: 144-52, 1992.

CHANDNANI , V.; HO, C.; GERHARTNER, J. MR findings in asymptomatic shoulders: a blind analysis using asymptomatic shoulders as controls. Clinical Orthopaedics, 16: 25-30, 1992.

CHARD, M. D.; HAZLEMAN, R.; HAZLEMAN, B. L. Shoulder disorders in the elderly: a community survey. Arthritis Rheum, 34 (6): 766-769, 1991.

COFIELD, R. H. Rotdor cuff disease of the shoulder. Jornal of bone and joint surgery. American volume, 67 (6): 974-9, 1985.

CONNOR, P. M.; BANKS, D. M.; TYSON, A. B.; COUMAS, J. S.; ALESSANDRO, D. F. Magnetic resonance imaging of the asymptomatic shoulder of overhead athletes. The American journal of sports medicine, 31(5), 724-727, 2003.

ELLENBECKER T. S.; ROETERT E. P.; BAILIE D. S.; DAVIES G. J.; BROWN S. W. Glenohumeral joint total rotation range of motion in elite tennis players and baseball pitchers. Med Sci Sports Exerc. Dec;34(12):2052-6, 2002

GARTSMAN, G. M.; BRINKER, M. R.; KAHN, M.; and KARAHAN, M. Self- assessment of the general health status in patients with five common shoulder conditions. J Shoulder Elbow Surg, 7(3): 228-237, 1998.

GOLDBERG, B. A.; MOWINSKI, R. J.; MATSEN, F. A.: Outcome of nonoperative management of full-thickeness rotator cuff tears. Clin Orthop, 382: 99-107, 2001.

GOLDBERG, B. A.; NOWINSKI, R. J.; and MATSEN, F. A. Outcome of nonoperative management of full-thickness rotator cuff tears. Clinical Orthopaedics, 382: 99-107, 2001. GOODMAN, R. S.: Abnormal findings on magnetic resonance images of asymptomatic shoulders. J Bone Joint Surg, 77A: 10-15, 1995.

GRANT, J. C. B.; and SMITH, C. G. Aging incidence of rupture of the the supraspinatus tendon. Anat Rec, 160: 666-669, 1948.

GREEN, S.; BUCHBINDER, R.; GLAZIER, R. Systematic review of randomized controlled trials of interventions for painful showder: selection criteria, outcome assessment and efficacy.

British Med J, 316: 354-360, 1998.

HARRYMAN, D. T.; HETTRICH, C. M.; SMITH, K. L.; CAMPBELL, B.; SIDLES, J. A.; MATSEN, F. A. Aprospective multpractice investigation of patientes with full-thickness rotator cuff tears.

Bone Joint Surg, 85a (4): 690-695, 2003.

HATTRUP, S. J. Rotator cuff rapair: relevance of patient age. J Soulder Elbow Surg, 4 (2): 95 100, 1995.

HAWKINS, R. H.; DUNLOP, R. Monoperative treatment of rotator cuff tears. Clin Orthop, 321: 178-188, 1995.

JOBE, F. W.; VANDERWILDE, R. S. Chronic shoulder problems in athletes. In Orthopaedic Knowledge Update: Sports Medicine, pp. 153-163. Edited, 153-163, Rosemont, IL, AOSSM and AAOS, 1994.

JOHANSSON, F. R.; SKILLGATE, E.; ADOLFSSON, A.; JENNER, G.; DEBRI, E.; SWARDH, L.; COOLS, A. M. Asymptomatic Elite Adolescent Tennis Players' Signs of Tendinosis in Their Dominant Shoulder Compared With Their Nondominant Shoulder. Journal of athletic training, 50(12), 1299- 1305, 2015.

JOST, B.; ZUMSTEIN, M.; PFIRRMANN, C. W. A.; ZANETTI, M.; GEBER, C. MRI Findings in Throwing Shoulders Abnormalities in Professional Handball Players.Clinical orthopaedics and related research, n. 434, pp. 130-137, 2005.

KEYES, E. L. Observations on rupture of the supraspinatus tendon based upon a study of seventy-three cadavers. Ann Surg, 97: 849-856, 1933. 
LEHMAN R. C. Shoulder pain in the competitive tennis player. Clin Sports Med 7: 309-327, 1988

LENZA, M.; BUCHBINDER, R.; TAKWOINGI, Y.; JOHNSTON, R. V.; HANCHARD, N. C.; FALOPPA, $F$. Magnetic resonance imaging, magnetic resonance arthrography and ultrasonography for assessing rotator cuff tears in people with shoulder pain for whom surgery is being considered. The Cochrane Library, 2013.

MANTONE, J. K.; BURKHEAD, W. Z.; NOONAN, J. Nonoperative treatment of rotator cuff tears. Orthop Clin North Am, 31 (2): 295-311, 2000.

MATSEN, F. A.; ARNTZ, C. T.; LIPPITT, S. B. Rotator cuff. In The Shoulder, pp. 755-839. Edited by ROCKWOOD, C. A.; MATSEN, F. A.; MICHEAL, A. W.; HARRYMAN, D. T., 755-839, Philadelphia, W. B. Saunders, 1998.

MCKEE, M. D. and YOO, D. J. The effect of surgery for rotator cuff disease on the general health status: Results of a prospective trial. J Bone Joint Surg, 82A(7): 970-979, 2000

MCMAHON, P. J.; PRASAD A.; FRANCIS, K. A. What is the prevalence of senior-athlete rotator cuff injuries and are they associated with pain and dysfunction? Clinical Orthopaedics and Related Research ${ }^{\circledR}$, 472(8), 2427-2432, 2014.

MILGROM, C.; SCHAFFLER, M.; GILBERT, S.; VANHOLSBEEK, M. Rotator-cuff changes in asymptomatic adults: the effect of age, hand dominance and gender. J Bone Joint Surg, 77B(2): 296-298, 1995.

MINIACI, A.; DOWDY, P. A.; WILLITIS, K. R.; VELLET, A. D. Magnetic resonance imaging evaluation of the rotator cuff tendons in the asymptomatic shoulder. Am J Sport Med, 23: 142-145, 1995.

MISAMORE, G. W.; ZIEGLER, D. W.; RUSHTON, J. L. Repair of the rotator cuff: a comparison of results in two populations of patientes. J bone Joint Surg, 77A(9): 1335-1339, 1995. MURRAY, T. F.;LAJTAI, G.; MILESKI, R. M. Arthoscopic repair of medium to large fullthickeness rotator cuff tears: autcome at 2- to 6-year follow-up. J Shoulder Elbow Surg, 11 (1): 19-24, 2002.

NOFFAL, G. J. Isokinetic Eccentric-to-Concentric Strength Ratios of the Shoulder Rotator Muscles in Throwers and Nonthrowers. The American Journal fo sports medicine, Vol. 31, No. 4: 537- 541, 2003.

OZAKI, J.; FUJIMOTO, S.; NAKAGAWA, Y.; MASUHARA, K.; TAMAI, S. Tears of the rotator cuff of the shoulder associated with pathological changes in the acromion: A study in cadavera. $J$ Bone Joint Surg Am, 70(8): 1224-30., 1988.

PRICKETT, W. D.; TEFFEY, S. A.; GALATZ, L. M. Accuracy of ultrasound imaging of the rotator cuff in shoulders that are painful postoperatively. J Bone Joint Surg, (85A): 1084-1089, 2003. RUOTOLO, C.; NOTTAGE, W. M. Surgical and nonsurgical management of rotator cuff tears. Arthroscopy, 18 (5): 527-531, 2002.

SHER, J. S.; URIBE, J. W.; POSADA, A.; MURPHY, B. J.; and ZATKIN, M. B. Abnormal findings on magnetic resonance images of assymptomatic shoulders. Clin Orthop, 304: 68-73, 1994. TIFFORD, C.; PLANCHER, K. Nonsurgical treatment of rotator cuff tears. In Orthopaedic Knowledge Update, Shoulder and Elbow, pp. 135-150. Edited, 135-150, Park Ridge, IL, AAOS, 1999.

TYTLERLEIGH-STRONG, G.; HIRAHARA, A.; MINIACl, A. Rotator cuff disease. Curr Opin Rheumatol, 13 (2): 135-145, 2001.

WATSON, E. M.; SONNABEND, D. H. Outcome of rotator cuff repair. J Shoulder Elbow Surg, 11 (3): 201-211, 2002. 
WIRTH, M. A.; BASAMANIA, C.; ROCKWOOD, C. A. Nonoperative management of fullthickness tears of the rotator cuff. Orthop Clin North Am, 28: 59-67, 1997.

YAMAGUCHI, K.; TETRO, M.; BLAM, O.; EVANOFF, B. A.; TEEFEY, S. A.; MIDDLETON, W. D. Natural history of asymptomatic rotator cuff tears: a longitudinal analysis of asymptomatic tears detected sonographically. J Shoulder Elbow SURG, 10 (3): 199-203, 2001.

ZANCA, G. G.; OLIVEIRA, A. B.; SACCOL, M. F.; EJNISMAN, B.; MATTIELLO-ROSA, S. M.

Functional torque ratios and torque curve analysis of shoulder rotations in overhead athletes with and without impingement symptoms. Journal of sports sciences. 29(15), 1603-1611, 2011. 
ANEXO 1 - DASH

\section{DISABILITIES OF THE ARM, SHOULDER AND HAND - British English}

Please rate your ability to do the following activities in the last week by circling the number below the appropriate response.

\begin{tabular}{|c|c|c|c|c|c|}
\hline & $\begin{array}{l}\text { NO } \\
\text { DIFFICULTY }\end{array}$ & $\begin{array}{l}\text { MILD } \\
\text { DIFFICULTY }\end{array}$ & $\begin{array}{l}\text { MODERATE } \\
\text { DIFFICULTY }\end{array}$ & $\begin{array}{l}\text { SEVERE } \\
\text { DIFFICULTY }\end{array}$ & UNABLE \\
\hline 1 Open a tight or new jar & 1 & 2 & 3 & 4 & 5 \\
\hline 2 Write & 1 & 2 & 3 & 4 & 5 \\
\hline 3 Turn a key & 1 & 2 & 3 & 4 & 5 \\
\hline 4 Prepare a meal. & 1 & 2 & 3 & 4 & 5 \\
\hline 5 Push open a heavy door & 1 & 2 & 3 & 4 & 5 \\
\hline $\begin{array}{l}6 \text { Place an object on a shelf above your } \\
\text { head }\end{array}$ & 1 & 2 & 3 & 4 & 5 \\
\hline $\begin{array}{l}7 \text { Do heavy household jobs (e.g. wash } \\
\text { windows, clean floors) }\end{array}$ & 1 & 2 & 3 & 4 & 5 \\
\hline 8 Garden or outdoor property work & 1 & 2 & 3 & 4 & 5 \\
\hline 9 Make a bed & 1 & 2 & 3 & 4 & 5 \\
\hline 10 Cary a shopping bag or briefcase & 1 & 2 & 3 & 4 & 5 \\
\hline 11 Carry a heavy object (over $10 \mathrm{lbs} / 5 \mathrm{kgs}$ ) & 1 & 2 & 3 & 4 & 5 \\
\hline 12 Change a lightbulb overhead & 1 & 2 & 3 & 4 & 5 \\
\hline 13 Wash or blow dry your hair & 1 & 2 & 3 & 4 & 5 \\
\hline 14 Wash your back & 1 & 2 & 3 & 4 & 5 \\
\hline 15 Put on a jumper & 1 & 2 & 3 & 4 & 5 \\
\hline 16 Use a knife to cut food & 1 & 2 & 3 & 4 & 5 \\
\hline $\begin{array}{l}17 \text { Recreational activities which require } \\
\text { little effort (e.g. card playing, knitting, etc) }\end{array}$ & 1 & 2 & 3 & 4 & 5 \\
\hline $\begin{array}{l}17 \text { Recreational activities which require } \\
\text { you to take some force or impact through } \\
\text { your arm, shoulder or hand (e.g. golf, } \\
\text { hammering, tennis etc) }\end{array}$ & 1 & 2 & 3 & 4 & $\mathbf{5}$ \\
\hline $\begin{array}{l}18 \text { Recreational activities in which you } \\
\text { move your arm freely (e.g. playing Frisbee, } \\
\text { badminton etc) }\end{array}$ & 1 & 2 & 3 & 4 & $\mathbf{5}$ \\
\hline $\begin{array}{l}20 \text { Manage transport needs (getting from } \\
\text { one place to another) }\end{array}$ & 1 & 2 & 3 & 4 & 5 \\
\hline 21 Sexual activities & 1 & 2 & 3 & 4 & $\mathbf{5}$ \\
\hline
\end{tabular}




\begin{tabular}{|c|c|c|c|c|c|}
\hline & $\begin{array}{l}\text { NOT AT } \\
\text { ALL }\end{array}$ & SLGHTLY & MODERATELY & $\begin{array}{c}\text { Quite a } \\
\text { bit }\end{array}$ & Extremely \\
\hline \multirow[t]{2}{*}{$\begin{array}{l}22 \text { During the past week, to what extent has } \\
\text { your arm, shoulder or hand problem } \\
\text { interfered with your normal social activities } \\
\text { with family. friends, neighbours or groups? } \\
\text { (circle number) }\end{array}$} & 1 & 2 & 3 & 4 & 5 \\
\hline & $\begin{array}{l}\text { NOT } \\
\text { LIMITED AT } \\
\text { ALL }\end{array}$ & $\begin{array}{l}\text { SLIGHTLY } \\
\text { LIMITED }\end{array}$ & $\begin{array}{l}\text { MODERATELY } \\
\text { LIMITED }\end{array}$ & $\begin{array}{l}\text { VERY } \\
\text { LMITED }\end{array}$ & UNABLE \\
\hline $\begin{array}{l}23 \text { During the past week, were you limited in } \\
\text { your work or other regular daily activities as } \\
\text { a result of your arm, shoulder or hand } \\
\text { problem? (circle number) }\end{array}$ & 1 & 2 & 3 & 4 & 5 \\
\hline $\begin{array}{l}\text { Please rate the severity of the following } \\
\text { symptoms in the last week (circle number) }\end{array}$ & NONE & MILD & MODERATE & SEVERE & EXTREME \\
\hline 24 Arm, shoulder or hand pain & 1 & 2 & 3 & 4 & 5 \\
\hline $\begin{array}{l}25 \text { Arm, shoulder or hand pain when you do } \\
\text { any specific activity }\end{array}$ & 1 & 2 & 3 & 4 & 5 \\
\hline $\begin{array}{l}26 \text { Tingling (pins and needles) in your arm, } \\
\text { shoulder or hand }\end{array}$ & 1 & 2 & 3 & 4 & 5 \\
\hline 27. Weakness in your arm, shoulder or hand & 1 & 2 & 3 & 4 & 5 \\
\hline \multirow[t]{2}{*}{28 Stiffness in your arm, shoulder or hand } & 1 & 2 & 3 & 4 & 5 \\
\hline & $\begin{array}{l}\text { NO } \\
\text { DIFFICULTY }\end{array}$ & $\begin{array}{c}\text { MILD } \\
\text { DIFFICULT } \\
y\end{array}$ & $\begin{array}{l}\text { MODERATE } \\
\text { DIFFCULTY }\end{array}$ & $\begin{array}{c}\text { SEVERE } \\
\text { DIFFCULT } \\
y\end{array}$ & $\begin{array}{c}\text { SO MUCH } \\
\text { DIFFICULTY } \\
\text { THATI } \\
\text { CAN'T SLEEP }\end{array}$ \\
\hline \multirow[t]{2}{*}{$\begin{array}{l}\text { have you had sleeping because of the pain in } \\
\text { your arm, shoulder or hand? (circle number) }\end{array}$} & 1 & 2 & 3 & 4 & 5 \\
\hline & $\begin{array}{l}\text { STRONGLY } \\
\text { DISAGREE }\end{array}$ & DISAGREE & $\begin{array}{l}\text { NEITHER AGREE } \\
\text { OR DISAGREE }\end{array}$ & AGREE & $\begin{array}{l}\text { STRONGLY } \\
\text { AGREE }\end{array}$ \\
\hline $\begin{array}{l}30 \text { I feel less capable, less confident or less } \\
\text { useful because of my arm, shoulder or hand } \\
\text { problem (circle number) }\end{array}$ & 1 & 2 & 3 & 4 & 5 \\
\hline
\end{tabular}

DASH DISABILTY/SYMPTOM SCORE = [(sum of $n$ responses) -1$]$ × 25 (where $n$ is the number of completed responses) n

A DASH score may not be calculated if there are greater than $\mathbf{3}$ missing items. 


\begin{tabular}{|c|c|c|c|c|c|}
\hline \multicolumn{6}{|c|}{$\begin{array}{l}\text { The following questions ask about the impact of your arm, shoulder or hand problem on your ability to work } \\
\text { (including home-making if that is your main work role). }\end{array}$} \\
\hline \multirow{2}{*}{\multicolumn{6}{|c|}{$\begin{array}{l}\text { Please indicate what your job / work is: } \\
\text { 口। do not work (you may skip this section). }\end{array}$}} \\
\hline & & & & & \\
\hline \multicolumn{6}{|c|}{ Please circle the number that best describes your physical ability in the past week. Did you have any difficulty: } \\
\hline & $\begin{array}{c}\text { NO } \\
\text { DIFFICULTY } \\
\end{array}$ & $\begin{array}{c}\text { MILD } \\
\text { DIFFICULTY }\end{array}$ & $\begin{array}{l}\text { MODERATE } \\
\text { DIFFICULTY }\end{array}$ & $\begin{array}{c}\text { SEVERE } \\
\text { DIFFICULTY }\end{array}$ & UNABLE \\
\hline 1. Doing your work in your usual way? & & & & & \\
\hline & 1 & 2 & 3 & 4 & 5 \\
\hline $\begin{array}{l}\text { 2. Doing your usual work because of arm, } \\
\text { shoulder or hand pain? }\end{array}$ & 1 & 2 & 3 & 4 & 5 \\
\hline $\begin{array}{l}\text { 3. Doing your work as well as you would } \\
\text { like? }\end{array}$ & 1 & 2 & 3 & 4 & 5 \\
\hline $\begin{array}{l}\text { 4. Spending your usual amount of time } \\
\text { doing your work? }\end{array}$ & 1 & 2 & 3 & 4 & 5 \\
\hline \multicolumn{6}{|c|}{$\begin{array}{l}\text { The following questions relate to the impact of your arm, shoulder or hand problem on playing your musical } \\
\text { instrument or sport or both. If you play more than one sport or instrument (or play both), please answer with } \\
\text { respect to that activity which is most important to you. }\end{array}$} \\
\hline \multicolumn{6}{|c|}{ 口I do not play a sport or an instrument. (You may skip this section). } \\
\hline Please circle the number that best describe & your physical & bility in the pa & st week. Did yo & u have any diff & iculty: \\
\hline & $\begin{array}{c}\text { NO } \\
\text { DIFFICULTY }\end{array}$ & $\begin{array}{c}\text { MILD } \\
\text { DIFFICULTY }\end{array}$ & $\begin{array}{l}\text { MODERATE } \\
\text { DIFFICULTY }\end{array}$ & $\begin{array}{c}\text { SEVERE } \\
\text { DIFFICULTY }\end{array}$ & UNABLE \\
\hline $\begin{array}{l}\text { 1. Playing your instrument or sport in your } \\
\text { usual way? }\end{array}$ & 1 & 2 & 3 & 4 & $\mathbf{5}$ \\
\hline $\begin{array}{l}\text { 2. Playing your musical instrument or } \\
\text { sport because of arm, shoulder or hand } \\
\text { pain? }\end{array}$ & 1 & 2 & 3 & 4 & $\mathbf{5}$ \\
\hline $\begin{array}{l}\text { 3. Playing your instrument or sport as well } \\
\text { as you would like? }\end{array}$ & 1 & 2 & 3 & 4 & $\mathbf{5}$ \\
\hline $\begin{array}{l}\text { 4. Spending your usual amount of time } \\
\text { practising or playing your instrument or } \\
\text { sport? }\end{array}$ & 1 & 2 & 3 & 4 & $\mathbf{5}$ \\
\hline
\end{tabular}

Scoring the optional modules: add up the assigned values for each response;

Divide by 4 (number of items); subtract 1; multiple by 25 .

An optional module score may not be calculated if there are any missing items.

ANEXO 2 - Escala Visual Analógica (EVA)

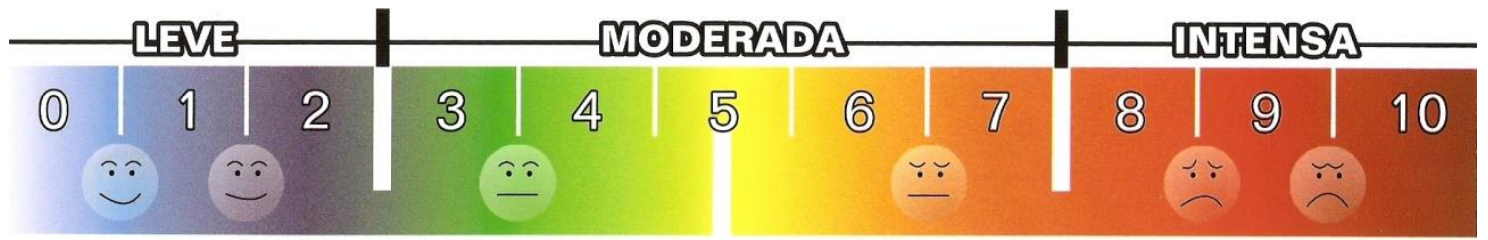

ESCALA VISUAL ANALÓGICA - EVA 
APÊNDICE A - Termo de consentimento

Centro Médico da Universidade de

Pittsburgh

Departamento de Cirurgia Ortopédica

Centro de Medicina Esportiva

Consentimento para atuar como participante em um estudo de pesquisa

TITLE: Rotator Cuff Tears: Characteristics Predicting Symptoms

INVESTIGADORA PRINCIPAL:

Dra.Tanya Hagen

Professora Assistente

Centro Médico da Universidade de

Pittsburgh

Divisão de Medicina Esportiva

3200 South Water Street

Pittsburgh, PA 15203

Telefone: 412-432-3641

\section{CO-INVESTIGADORES:}

Dr. Moacir Silva Neto

Kimberly Francis, MS, MPA

Pesquisador Médico

Coordenadora de Pesquisas

Centro Médico da Universidade de Clínicas

Pittsburgh

Divisão de Medicina Esportiva

Centro Médico da Universidade de Pittsburgh

Divisão de Medicina Esportiva

3200 South Water Street

Pittsburgh, PA 15203

3200 South Water Street

Phone: 412-432-3641

Pittsburgh, PA 15203

Phone: 412-432-3721

FONTE DE SUPORTE: Departamento de Cirurgia Ortopédica

\section{Por que este estudo esta sendo realizado?}

O propósito deste estudo é comparar características de indivíduos com três condições diferentes do ombro: ombros normais, ombros sem dor com rupturas do manguito rotador (MR) e ombros doloridos com rupturas do MR. Nós iremos determinar: 
- Se os indivíduos têm ombros sem dor funcionando normalmente ou se simplesmente aprenderam a lidar com a disfunção do ombro sem dor;

- $\quad$ Se indivíduos com rupturas do MR com o ombro assintomático apresentam diferenças no arco de movimento, força e pontuações de medidas funcionais quando comparados com indivíduos que têm um ombro com o MR intacto e sem dor ou com rupturas de MR e dor ao mesmo tempo;

- $\quad$ Se existe correlação entre alguns tipos de exercícios e dor no ombro, função e lesão.

\section{Quem está sendo convidado para participar neste estudo de pesquisa?}

Todos os atletas de 18 anos ou acima de 18 anos que estejam participando dos jogos Pan-Americanos de 2007 serão convidados a participar deste estudo.

\section{Quais são os procedimentos deste estudo?}

\section{Determinando a presença ou ausência de Dor no Ombro}

Você será selecionado para o grupo com dor ou sem dor baseado em sua pontuação na Escala Analógica da Dor (EAD). Você vai marcar a quantidade de dor que você está experimentando em uma linha de $100 \mathrm{~mm}$. (3 minutos para completar).

Determinando a presença ou ausência de um Manguito Rotador intacto usando Ultrassom

Cada participante do estudo passará por um exame de ultrassom do ombro. Todos os exames serão realizados na Poli-Clínica (Centro Médico) na Vila Pan-americana. Se houver rupturas do MR, o tamanho da lesão será medido. Você estará sentado com seu ombro ao seu lado. Um gel condutivo hipo-alergênico e não tóxico será aplicado ao seu ombro. $O$ transdutor do Ultrassom (que tem o tamanho de uma lanterna pequena) será esfregado levemente no seu ombro para frente e para trás. Ondas de ultrassom são emitidas pelo transdutor e uma imagem é gerada. Pode ser que você seja solicitado a mexer seu ombro para melhorar a qualidade da imagem. (10 minutos para ser completado)

Disfunções do Braço, Ombro e Mão (DASH).

A Medida do Desfecho Clínico DASH avalia disfunção e sintomas relacionados distúrbios musculares e esqueléticos do braço, ombro e mão. O questionário de 30 perguntas inclui 21 itens sobre função física, 6 itens sobre sintomas e 3 itens sobre função social. Adicionalmente, existem dois módulos opcionais: um direcionado para atletas e artistas que realizam apresentações em palcos e outro para populações trabalhadoras. (5 minutos para ser completado)

\section{Arco de Movimento (ADM) e Teste de Força}

Testes ativos, passivos e resistidos serão realizados por um fisioterapeuta. $\mathrm{O}$ arco de movimento dos seus ombros será medido com um goniômetro (um instrumento na forma de um arco usado para medir ângulos). O limite até o qual você pode elevar e/ou rodar seus braços será medido usando o goniômetro. Você realizará este teste estando deitado em suas costas em uma mesa de exame e quando estiver em pé. Um dinamômetro (instrumento usado 
para medir força) será usado para medir a força do seu ombro. Você irá gerar força contra o dinamômetro com seu braço em diferentes posições (20 minutos para ser completado)

\section{Quais são os possíveis riscos e desconfortos deste estudo?}

Para assegurar que todos os indivíduos estejam a salvos do ponto de vista médico e que não haja contra indicação para a realização dos procedimentos associados ao estudo, todos os participantes serão avaliados por um médico antes de ser recrutado para o estudo. $\mathrm{Na}$ eventualidade do participante se lesionar durante os testes, ou procedimentos de treinamento, o médico do participante será informado o mais rápido possível. Existem salas médicas localizadas no local onde os testes serão realizados. Caso a lesão requeira atenção médica imediata, um médico no local será notificado. Adicionalmente, qualquer evento adverso que por ventura ocorra associado ao estudo será reportado ao Comitê de Revisão Institucional da Universidade de Pittsburgh.

$O$ teste de $A D M$ e força propostos são similares ao que se realize rotineiramente em um exame clínico do ombro quando um paciente esta sendo avaliado para uma lesão do manguito rotador. $\mathrm{O}$ risco mais significativo associado a estes testes é sentir o músculo dolorido. O potencial para os indivíduos sentirem um músculo dolorido temporariamente ou um desconforto mínimo no ombro devido às medidas de arco de movimento e força são comuns ( $1 \%$ a $25 \%$ das pessoas; 1 a 25 pessoas em 100 ).

O exame de ultrassom não envolve o uso de radiação ionizante ou de contrastes injetáveis e é considerado um procedimento que não apresenta nenhum risco 'a saúde conhecido. Não existe dor física associada a este procedimento. Os gels comumente usados não geram nenhum efeito adverso conhecido.

É esperado que você não sofra nenhum efeito físico ou psicológico adverso como conseqüência de completar os questionários. Quando é obtida informação médica para fins de pesquisas, existe o risco potencial de se haver quebra de confidencialidade.

\section{Eu me beneficiarei por participar deste estudo?}

A participação neste estudo não oferece nenhum benefício direto. Dentre os benefícios potenciais está incluído o conhecimento da integridade do manguito rotador nos indivíduos assintomáticos. Os resultados do estudo podem levar a um refinamento da reabilitação de ombro em casos de rotura do MR e auxiliar o desenvolvimento de estratégias preventivas.

Eu ou o meu seguro de saúde ou seremos cobrados pelos custos de qualquer procedimento realizado como parte deste estudo de pesquisa?

Nem você, nem seu seguro de saúde serão cobrados pelos custos de qualquer procedimento a ser realizado para os propósitos deste estudo de pesquisa.

\section{Eu serei pago se participar neste estudo de pesquisa?}

Você não será pago para participar deste estudo de pesquisa.

\section{Quem pagará caso eu me machuque como resultado da minha participação neste estudo?}

Os pesquisadores da Universidade de Pittsburgh e seus associados que prestam serviços na UPMC reconhecem a importância da sua participação voluntária neste estudo de 
pesquisa. Essas pessoas e sua equipe farão tudo que for possível para minimizar, controlar e tratar qualquer lesão que possa surgir como resultado desta pesquisa. Se você julgar que foi lesionado como resultado dos procedimentos desta pesquisa, os quais estão sendo informados, favor contactar imediatamente o investigador principal ou um dos coinvestigadores listados na primeira página deste documento.

Tratamento médico de emergência para lesões relacionadas à sua participação neste estudo de pesquisa será fornecido no Hospital Barra D'Or, Hospital Copa D'Or e Hospital QuintaD'Or. É possível que o hospital cobre o seu seguro pelo custo do tratamento de emergência, mas nenhum desses custos será cobrado diretamente de você. Caso sua lesão relacionada ao estudo requeira tratamento médico além do tratamento de emergência, você será responsável pelo custo deste tratamento de seguimento a não ser que seja especificamente listado abaixo. Não existem planos para compensação monetária. Todavia, você não estará abrindo mão de qualquer direito legal ao assinar este documento.

\section{Quem irá ter conhecimento da minha participação neste estudo de pesquisa?}

Qualquer informação sobre você obtida a partir desta pesquisa será guardada como confidencial (privada), o máximo possível. Tudo que for registrado relacionado ao seu envolvimento nesta pesquisa será guardado em uma cabine trancada de armazenar arquivos. Sua identidade nesses itens registrados será indicada por um número de caso e não por seu nome. Qualquer informação que faça a ligação deste número de caso à sua identidade será guardada separada dos dados da pesquisa. Você não será identificado por seu nome em nenhuma publicação dos resultados da pesquisa, a não ser que você assine um documento de consentimento separado dando esta permissão (liberação).

\section{A minha participação neste estudo de pesquisa é voluntária?}

Sua participação neste estudo de pesquisa, para incluir o uso e exposição da sua informação identificável para os propósitos descritos acima, é completamente voluntária. (Note, entretanto, que se você não fornecer seu consentimento para o uso e exposição da sua informação identificável para os propósitos descritos acima, não será permitida a sua participação neste estudo de pesquisa.) $O$ fato de você fornecer ou não o seu consentimento para participação neste estudo de pesquisa não terá nenhuma influência no seu relacionamento atual ou futuro com a Universidade de Pittsburgh. $O$ fato de você fornecer ou não seu consentimento não terá nenhuma influência no seu atendimento médico atual ou futuro em um hospital da UPMC ou prestador de serviços de saúde afiliados ou no seu relacionamento com o seu prestador de seguro de saúde atual.

Eu posso retirar, em uma data futura, meu consentimento para participar neste estudo de pesquisa?

Você pode retirar, a qualquer momento, seu consentimento para participação em estudos de pesquisa, para incluir o uso e exposição da sua informação identificável para os propósitos descritos acima. (Note, entretanto, que se você retirar seu consentimento para o uso e exposição dos seus dados médicos registrados para os propósitos descritos acima, você também será excluído, de uma forma geral, de uma participação futura neste estudo de pesquisa). Qualquer informação médica ou de pesquisa identificável registrada para, ou como resultado da sua participação neste estudo anterior à data da retirada formal do consentimento, poderá ser usada e exposta pelos investigadores para os propósitos descritos acima. 
Para retirar formalmente o seu consentimento de participação neste estudo de pesquisa, você deve providenciar uma notificação escrita datada a respeito dessa decisão para o investigador principal deste estudo de pesquisa no endereço descrito na primeira página deste formulário.

Sua decisão de retirar seu consentimento em participar neste estudo de pesquisa não irá ter nenhum efeito no seu relacionamento presente ou futuro com a Universidade de Pittsburgh. Sua decisão de retirar seu consentimento para participar neste estudo não terá nenhum efeito no cuidado médico presente ou futuro em qualquer hospital UPMC ou prestadores de serviços de saúde afiliados, nem com o relacionamento futuro ou presente com seu provedor de seguro de saúde.

\section{Como eu posso obter mais informação sobre este estudo?}

Se você tiver qualquer dúvida futura sobre este estudo de pesquisa, você pode contactar os investigadores listados no início deste formulário de consentimento. Se você tem alguma pergunta sobre seus direitos como uma pessoa que esta sendo pesquisada, por favor, entre em contato com o Advogado de Proteção a Indivíduos Humanos na Universidade de Pittsburgh no escritório da IRB, 00-1-866-212-2668.

\section{CONSENTIMENTO VOLUNTÁRIO}

As informações acima me foram explicadas e todas minhas perguntas até este momento foram respondidas. Entendo que sou incentivado(a) a fazer perguntas sobre qualquer aspecto deste estudo de pesquisa durante o curso deste estudo, e que qualquer pergunta futura será respondida por um indivíduo qualificado ou pelo(s) investigador(es) listados na primeira página deste documento de consentimento pelos números de telefone fornecidos. Entendo que sempre poderei exigir que minhas perguntas, receios e reclamações sejam abordados pelos investigadores assinalados.

Entendo que posso contactar o Advogado de proteção a Indivíduos Humanos do escritório da IRB, Universidade de Pittsburgh (00-1-866-212-2668) para discutir problemas, receios e perguntas; receber informação; oferecer contribuições; ou discutir situações durante o evento durante as quais a equipe de pesquisa não esteja disponível.

Ao assinar este formulário, concordo em participar deste estudo de pesquisa. Uma cópia deste formulário de consentimento me será dada.

Nome do Participante por Extenso

Assinatura do Participante

Data 
Eu certifico que forneci explicação sobre a natureza e propósito deste estudo de pesquisa ao(s) indivíduo(s) descrito(s) acima, e que dialoguei sobre os potenciais benefícios e possíveis riscos de se participar deste estudo.

Todas as perguntas que o indivíduo teve a respeito desse estudo foram respondidas e sempre estaremos disponíveis para abordar qualquer pergunta à medida que elas apareçam.

$\overline{\text { Nome por Extenso da Pessoa Recebendo o Consentimento Função no Estudo }}$

Assinatura da Pessoa Recebendo o Consentimento

Data 
APÊNDICE B - termo de aprovação do conselho de ética da Universidade de Pittsburgh

\author{
pitt seal
University of Pittsburgh Institutional Review Board

\section{Memorandum} \\ To: TANYA HAGEN \\ From: SUE BEERS PHD, Vice Chair \\ Date: $\quad 7 / 12 / 2007$ \\ IRB\#: PR007060028 \\ Subject: Rotator Cuff Tears: Characteristics Predicting Symptoms
}

3500 Fith Avenue Ground Level Pittsburgh, PA 15213 (412) $383-1480$ (412) 383-1508 (fax)

Your research study has received expedited review and approval from the Institutional Review Board under 45 CFR 110.(4) data collection/noninvasive , 45 CFR 110.(7) characteristics/behaviors.

Please note that the advertisement that was submitted for review has been approved as written.

The waiver for the requirement to obtain a written informed consent for screening has been approved.

Please note the following information:
Approval Date: $7 / 12 / 2007$
Expiration Date: $7 / 11 / 2008$

Please note that it is the investigator's responsibility to report to the IRB any unanticipated problems involving risks to subjects or others [see 45 CFR 46.103(b)(5) and 21 CFR 56.108 (b)]. The IRB Reference Manual (Chapter 3, Section 3.3) describes the reporting requirements for unanticipated problems which include, but are not limited to, adverse events. If you have any questions about this process, please contact the Adverse Events Coordinator at $412-383-1480$.

The protocol and consent forms, along with a brief progress report must be resubmitted at least one month prior to the renewal date noted above as required by FWA00006790 (University of Pittsburgh), FWA00006735 (University of Pittsburgh Medical Center), FWA00000600 (Children's Hospital of Pittsburgh), FWA00003567 (Magee-Womens Health Corporation), FWA00003338 (University of Pittsburgh Medical Center Cancer Institute).

Please be advised that your research study may be audited periodically by the University of Pittsburgh Research Conduct and Compliance Office. 